\title{
étude de la stabilité des rives de la cuvette du barrage idriss 1 er au maroc
}

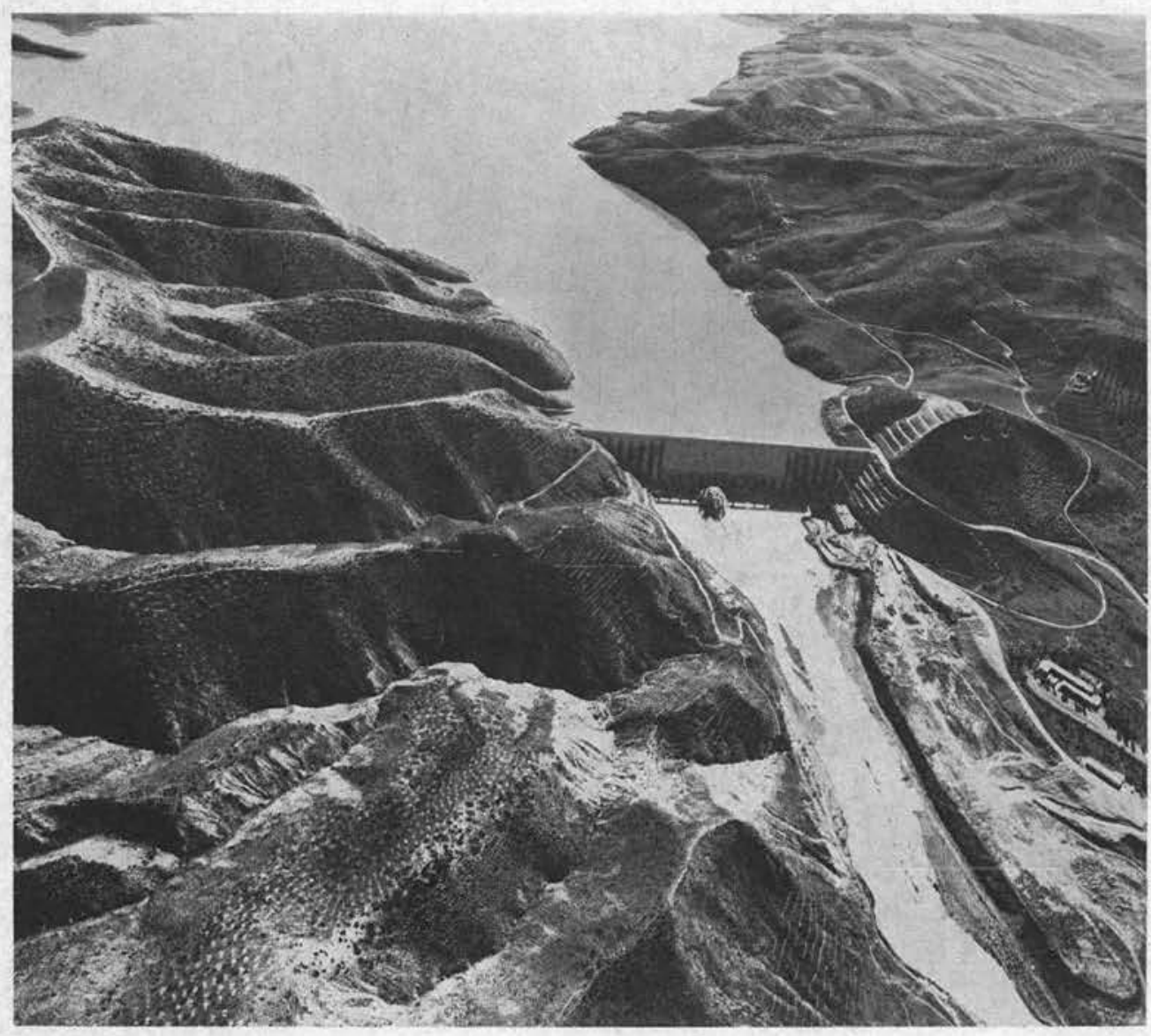

(Photo "Atlas-Aviation ", Rabat).

\section{G. L'Hériteau}

Président-Directeur Général Mécasol, Paris

\section{Moudden}

Directeur de l'Equipement Direction de l'Hydraulique - Rabat

\section{G. Post}

\section{Directeur}

Coyne \& Bellier, Paris 


\section{ETUDE DE LA STABILITE DES RIVES DE LA CUVETTE DU BARRAGE IDRISS-I AU MAROC}

Le barrage Idriss- $\left.\right|^{\text {er }}$ a été réalisé par la Direction de l'Hydraulique du ministère des Travaux Publics et des Communications du Royaume du Maroc. C'est un barrage à contreforts de $72 \mathrm{~m}$ de hauteur. créant sur l'oued Inaouène un important réservoir pouvant garantir l'irrigation de plus de 100000 ha. Cet ouvrage, mis en eau en 1973, est exploité dans une première phase à une cote basse (plus hautes eaux à 206) et le sera, dans une seconde phase, après dérivation des eaux du Haut Sebou, à la cote finale (plus hautes eaux à 220,30 ).

Les volumes accumulés sont très importants : $750 \mathrm{Mm}^{3}$ à la cote 206 et $1430 \mathrm{Mm}^{3}$ à la cote 220,30 et les eaux baignent plus de $130 \mathrm{~km}$ de côtes situées dans des terrains médiocres pouvant subir des marnages de 30 à $35 \mathrm{~m}$ en exploitation normale et de $50 \mathrm{~m}$ environ en cas de vidange totale.

II se posait donc un important problème de stabilité des rives de la cuvette qui fait l'objet du présent exposé.

\section{STABILITY DESIGN OF THE RESERVOIR BANK OF IDRISS $1^{\text {rst }}$ DAM IN MOROCCO}

Idriss 1st dam was constructed by the Direction of Hydraulic (Ministry of Public Works and Transports) of Morocco. It is a buttress dam of $72 \mathrm{~m}$ height on the Inaouene wadi. It forms an important reservoir allowing the irrigation of more than 100000 hectares.

The reservoir, filled in 1973, is under operation for the first stage at a low level (highest water at elevation 206) and for the second stage -after diversion of Upper Sebou course - will be at the final level (highest water at elevation 220.30).

The total volumes are very large: $750 \mathrm{Mm}^{3}$ at elevation 206 and $1.430 \mathrm{Mm}^{2}$ at elevation 220.30. The reservoir water level can vary up to $30-35 \mathrm{~m}$ during normal operating conditions, and about $50 \mathrm{~m}$ for total drawdown. These waters interest more than $130 \mathrm{~km}$ of slopes developed in low characteristics grounds.

The major reservoir slope stability problem is the object of the paper which examines:

- the detection of potential sliding areas;

- the nature and characteristics of the encountered materials;

- the slope stability computations;

- the measures taken to avoid sliding in areas where they should be damaging:

- the surveying performed three years after the filling of the reservoir at the level of the first stage. 


\title{
étude de la stabilité des rives de la cuvette du barrage idriss $1^{\text {er }}$ au maroc
}

\author{
par G. L'HÉRITEAU, M. MOUDDEN et G. POST
}

\section{OBJET DE L'ETUDE}

Il s'agissait de détecter les zones de glissement préexistantes ou potentielles, dont la mise en mouvement pourrait avoir des conséquences sur :

- la sécurité du barrage (effet d'une vague consécutive à un glissement) et la capacité de la retenue (conséquence économique d'une réduction de la capacité utile);

- la sécurité des douars à proximité du plan d'eau (douars à déplacer) ;

- les terres cultivées ou cultivables (à exproprier) ;

- les voies de communications ferrées et routières (à déplacer ou conforter).

Cette étude a comporté (fig. 1) :

- l'inventaire des zones glissées ou susceptibles de glisser ;

- l'étude plus approfondie de certaines zones critiques, soit proches du barrage (zones 1 et 2 ), soit intéressant la stabilité de la voie ferrée lors du remplissage de première phase de la retenue (zones 6 et 7), cette dernière n'étant déplacée que lors de l'exploitation de $2^{\mathrm{c}}$ phase (fig. 1).

La méthodologie employée a donc comporté deux phases successives :

1) une étude géologique générale sur photos aériennes (au $1 / 15000$ ) avec levé au $1 / 20000$, complétée par une étude statistique des pentes et des hauteurs des versants et repérage des glissements préexistants et des zones les plus menaçantes;

2) une étude géologique détaillée $(1 / 2000)$ des zones les plus critiques avec exécution de forages, tranchées et puits et essais géotechniques (in situ et en laboratoire).

Les études géologiques ont été effectuées avec le concours du Professeur R. Barbier.

Fig. 1.

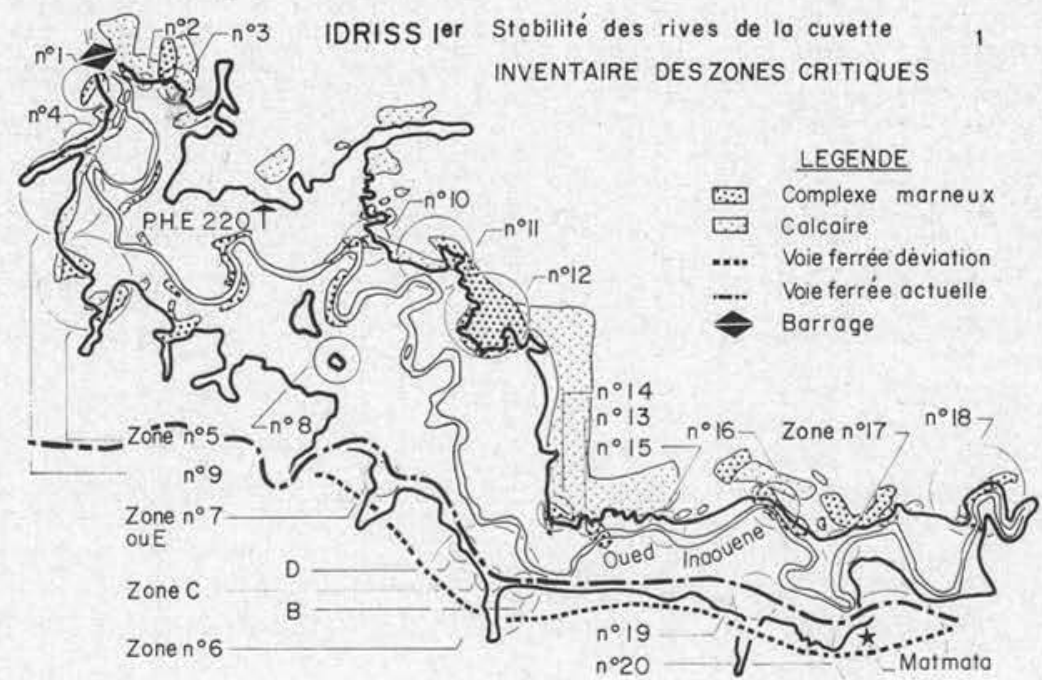

\section{CADRE GEOLOGIQUE REGIONAL}

La région intéressée s'intègre dans la chaîne rifaine et plus précisément dans l'unité prérifaine de cette chaîne. Cette région soumise à de violents efforts tangentiels lors de la surrection de la chaîne a été marquée par de nombreux et importants décollements au niveau de couches épaisses, très plastiques, rapportées princi- palement au Trias et au Crétacé inférieur marneux et salifère.

C'est au cours du Crétacé et du Tertiaire que se sont produits ces mouvements qui donnent au Prérif son aspect géologique fait d'écailles calcaires constituani les reliefs fichés dans une pâte marneuse broyé. appelée le " complexe marneux ». 


\section{LES TERRAINS ET LEURS CARACTERISTIQUES}

Seront étudiées successivement les deux grandes formations rencontrées : le complexe marneux d'une part et les circulaires d'autre part; sans insister sur les formations récentes plus ou moins grossières parfois cimentées, recouvrant ces formations sur 0.50 à 10 ou $15 \mathrm{~m}$

\subsection{Le complexe marneux et les marnes miocènes}

Le " complexe " définit un ensemble de matériaux plastiques ayant subi les chevauchements et les glissements de masses plus rigides. Géologiquement parlant, ce terme ne possède aucun sens car il réunit dans un même ensemble des formations de nature et d'âges très différents. Cependant, ces matériaux sont si souvent mêlés et malaxés qu'ils constituent une entité aux caractéristiques mécaniques extrêmement constantes dans leur médiocrité.

Ce sont des marnes gypsifères versicolores, bariolées ou noires, du trias ou crétacé, présentant des indices de plasticité toujours supérieurs à $30 \%$, des poids volumiques de l'ordre de 16 à $17.5 \mathrm{kN} / \mathrm{m}^{3}$, une cohésion moyenne en essai non consolidé non drainé de $100 \mathrm{kN} / \mathrm{m}^{2}$.

Les essais triaxiaux consolidés drainés effectués sur échantillons intacts par le Laboratoire Public d'Essais et d'Etudes de Casablanca ont donné les valeurs moyennes suivantes :

$$
\begin{aligned}
& \mathrm{C}^{\prime}=60 \mathrm{kN} / \mathrm{m}^{2} \\
& \Phi^{\prime}=17^{\circ}
\end{aligned}
$$

pour des couches profondes (pressions de terrain supérieures à la pression de gonflement voisine de $200 \mathrm{kN} / \mathrm{m}^{2}$ ).

Les essais effectués sur les marnes miocènes ont révélé également des indices de plasticité compris entre 30 et $50 \%$. Ce sont des matériaux surconsolidés, fissurés (poids volumique 17,5 à $19 \mathrm{kN} / \mathrm{m}^{3}$ ) dont l'angle de cisaillement effectif varie entre 23 et $25^{\circ}$ (pour des déformations de 6 à $8 \%$ ).

\section{REPARTITION ET STABILITE DES TERRAINS DE LA CUVETTE}

\subsection{Répartition des terrains Etude statistique des pentes}

La cuvette est marquée par une différence de morphologie nette entre les deux rives : la rive gauche est plate dans l'ensemble, trahissant un substratum marneux: la rive droite présente un certain nombre de reliefs plus accentués, peu élevés, formés de calcaires marneux, séparés par des collines moins élevées, très ravinées et constituées de marnes. Cette différence tient vraisemblablement au fait que les deux rives appartiennent à deux régions géologiques de l'unité prérifaine.

L'étude statistique des pentes et des hauteurs des versants est traduite par les figures 2 à 5 et condensée dans le tableau 1.

\subsection{Répartition des terrains}

Sur $134 \mathrm{~km}$ de versant, $83 \%$ sont constitués de marnes (affleurant ou sous faible recouvrement quaternaire) et le reste de calcaires ou calcaires marneux.

\subsection{Répartition des pentes} à $20^{\circ}$. longueur développée de $20 \mathrm{~km}$.

\subsection{Hauteur des versants} l'appui rive droite du barrage.

\subsection{Zones glissées ou écroulées}

La résistance résiduelle de ces différentes marnes semble comprise entre 15 et $20^{\circ}$.

\subsection{Les calcaires constituant les écailles}

Dans l'écaille d'Arabat, sur laquelle se situe le barrage, on note de bas en haut, surmontant le complexe marneux :

- des calcaires à silex ;

- des calcaires marneux dits « calcaires du site ";

- une série gréseuse.

Ces faciès changent peu et se retrouvent dans les autres écailles de la cuvette; toutefois, ils deviennent plus marneux par variation latérale de faciès.

Les «Calcaires du site " sont les seuls à avoir été étudiés in situ par le laboratoire (LPEE) de Casablanca car ils constituent la fondation du barrage, études qui consistent en :

- des essais de chargement au vérin sur disque rigide ;

- des essais de cisaillement in situ dans la masse et sur joints stratigraphiques ;

des essais de cisaillement directs sur carottes précoupées ou le long de joints.

Les caractéristiques de rupture seraient (pour les contraintes normales égales ou inférieures à 20 bars) :

- pour le calcaire homogène fracturé en larges mailles :

$$
\mathrm{C}=500 \text { à } 1000 \mathrm{kN} / \mathrm{m}^{2} \quad \Phi=36^{\circ} \text { à } 43^{\circ}
$$

- pour un calcaire assez sain avec un plan de rupture passant selon un joint stratigraphique sans remplissage argileux :

$$
\mathrm{C}=50 \text { à } 350 \mathrm{kN} / \mathrm{m}^{2} \quad \Phi=32^{\circ} \text { à } 36^{\circ}
$$

- pour un calcaire décomprimé et localement broyé avec remplissage argileux ou joint marneux :

$$
\mathrm{C}=0 \text { à } 50 \mathrm{kN} / \mathrm{m}^{2} \quad \Phi=20^{\circ} \text { à } 24^{\circ}
$$

Plus de la moitié des versants ont une pente inférieure à $10^{\circ}$ et $12 \%$ seulement une pente supérieure

Les versants constitués de marnes ont presque tous une pente inférieure à $20^{\circ}$ et seulement $15 \%$ ont une pente supérieure à $15^{\circ}$, ce qui correspond à une

Les hauteurs des versants sont assez faibles; le maximum, $250 \mathrm{~m}$, est dû à l'éperon calcaire constituant

$80 \%$ des versants ont une hauteur inférieure à $150 \mathrm{~m}$ et $45 \%$ une hauteur inférieure à $100 \mathrm{~m}$.

Le tableau 1 montre que les zones glissées dans les marnes, superficielles ou profondes, mais généralement 
IDRISS $\left.\right|^{\text {er }}$ Stabilité des rives de la cuvette

TABLEAU । - REPARTITION DES PENTES EN FONCTION

DE LA NATURE DU MATERIAU

(longueur développée ò lo cote 220,30 P.H.E)

\begin{tabular}{|c|c|c|c|c|c|}
\hline & $<10^{\circ}$ & $10^{\circ} \mathrm{d} 15^{\circ}$ & $15^{\circ}$ à $20^{\circ}$ & $>20^{\circ}$ & TOTAL \\
\hline Longueur totale des rives en $\mathrm{km}$. & $\begin{array}{l}70 \mathrm{~km} \\
52 \%\end{array}$ & $\begin{array}{l}29 \mathrm{~km} \\
22 \%\end{array}$ & $\begin{array}{l}19 \mathrm{~km} \\
14 \%\end{array}$ & $\begin{array}{l}16 \mathrm{~km} \\
12 \%\end{array}$ & $\begin{array}{l}134 \mathrm{~km} \\
100 \%\end{array}$ \\
\hline $\begin{array}{l}\text { Longueur des zones dans } \\
\text { les calcaires }\end{array}$ & $0 \%$ & $\begin{array}{l}0 \\
0 \%\end{array}$ & $\begin{array}{l}4 \mathrm{~km} \\
3 \%\end{array}$ & $\begin{array}{l}19 \mathrm{~km} \\
14 \%\end{array}$ & $\begin{array}{l}23 \mathrm{~km} \\
17 \%\end{array}$ \\
\hline $\begin{array}{l}\text { Longueur des zones dons } \\
\text { les marnes }\end{array}$ & $\begin{array}{l}65 \mathrm{~km} \\
48 \%\end{array}$ & $\begin{array}{l}26 \mathrm{~km} \\
20 \%\end{array}$ & $\begin{array}{l}16 \mathrm{~km} \\
12 \%\end{array}$ & $\begin{array}{l}4 \mathrm{~km} \\
3 \%\end{array}$ & $\begin{array}{l}111 \mathrm{~km} \\
83 \%\end{array}$ \\
\hline $\begin{array}{l}\text { Longueur des zones } \\
\text { colcaires éboulèes }\end{array}$ & $\begin{array}{l}0 \\
0 \%\end{array}$ & $\begin{array}{l}0 \\
0 \%\end{array}$ & $0 \%$ & $0,5 \mathrm{~km}$ & $\begin{array}{l}0,5 \mathrm{~km} \\
0,4 \%\end{array}$ \\
\hline $\begin{array}{l}\text { Longueur des zones glissées } \\
\text { (superficielles et profondes) } \\
\text { dans le complexe marneux }\end{array}$ & $\begin{array}{l}0 \\
0 \%\end{array}$ & $\begin{array}{l}1,8 \mathrm{~km} \\
1,3 \%\end{array}$ & $\begin{array}{c}4,5 \mathrm{~km} . \\
3,4 \%\end{array}$ & $\begin{array}{l}3 \mathrm{~km} \\
2,2 \%\end{array}$ & $\begin{array}{l}9,3 \mathrm{~km} \\
6,9 \%\end{array}$ \\
\hline
\end{tabular}

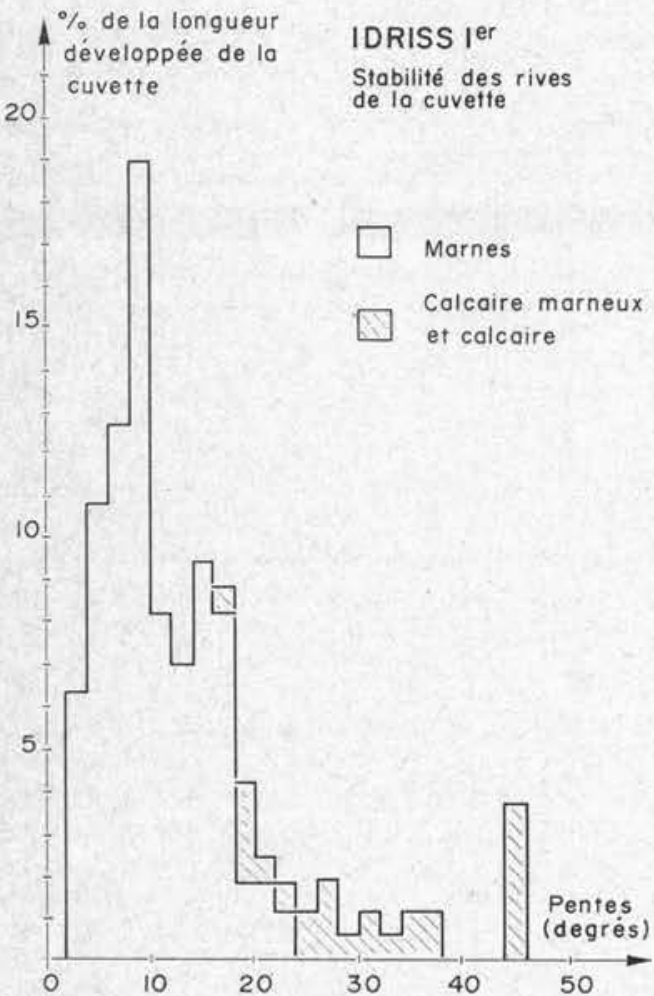

Fig. 2.

Fig. 4.
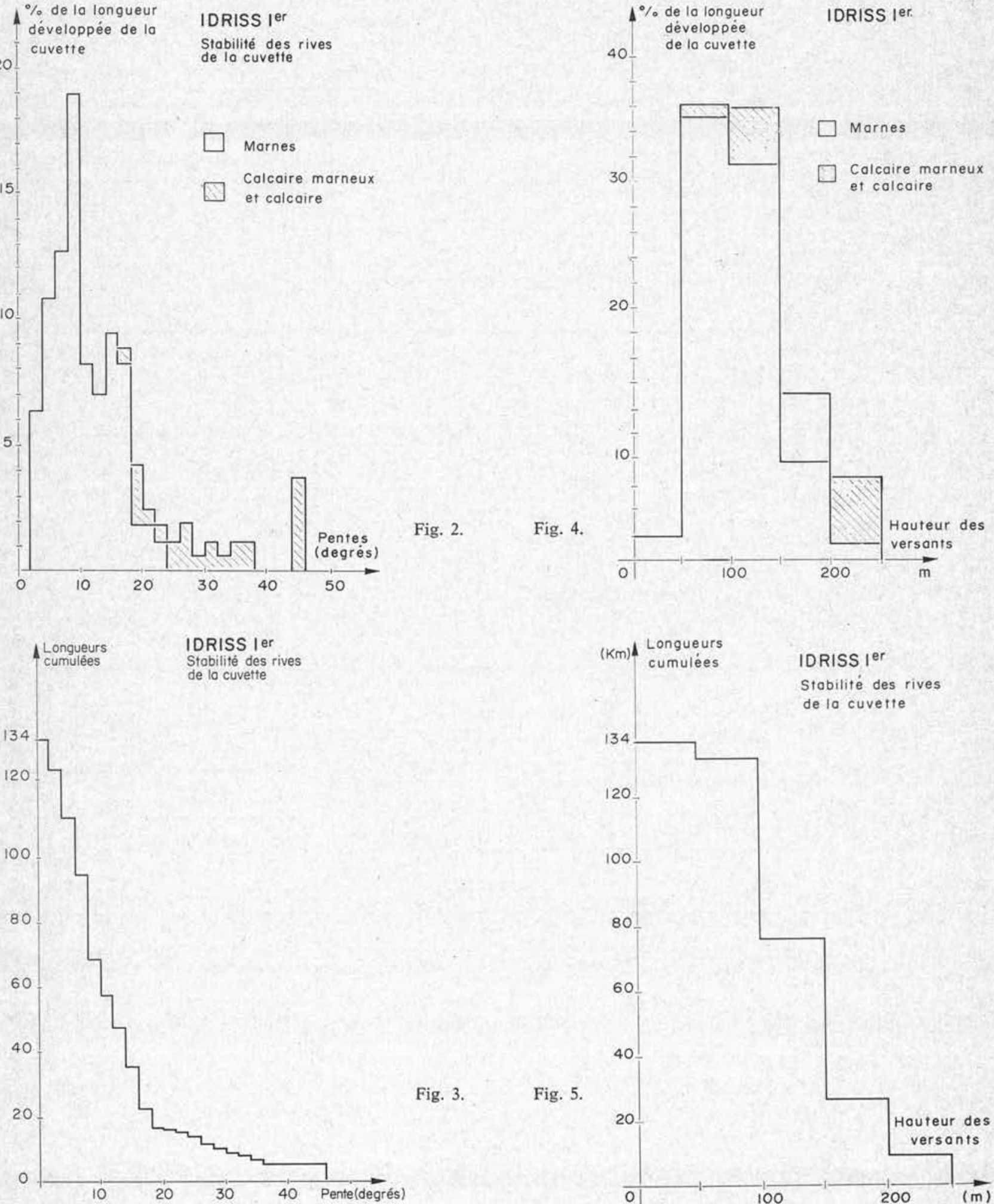


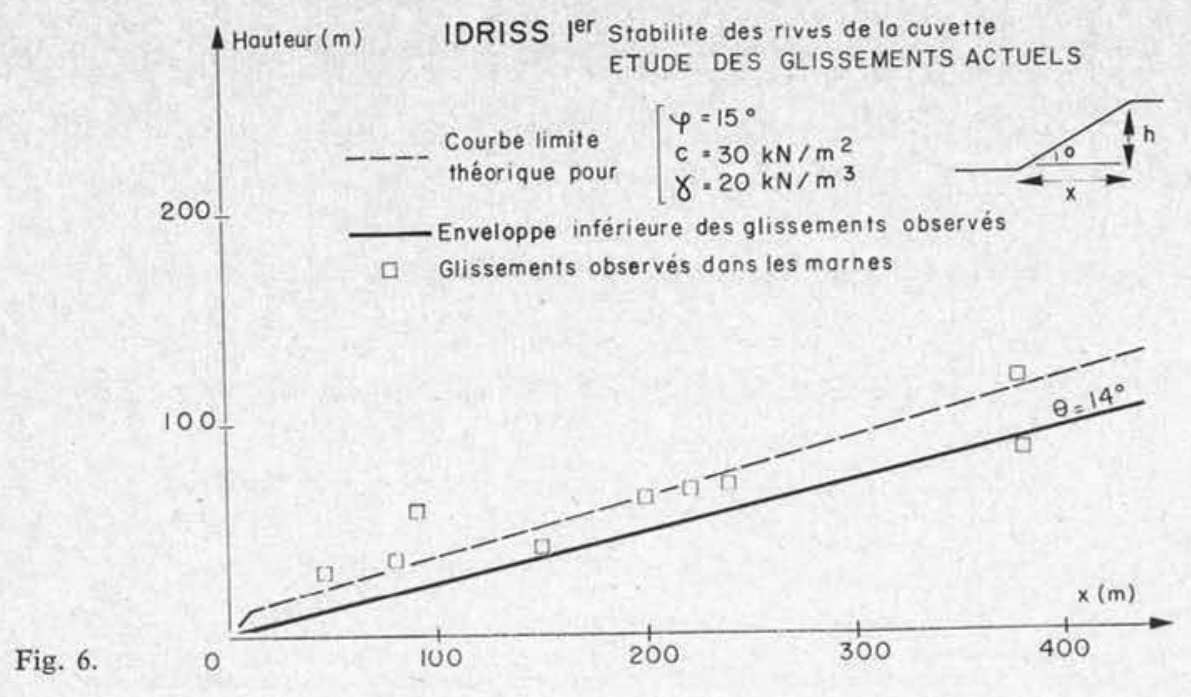

peu volumineuses, représentent environ $8.5 \%$ de la longueur développée des versants marneux et près de $50 \%$ de la longueur développée des rives marneuses pentées à plus de $15^{\circ}$.

Les écroulements dans les calcaires sont très peu nombreux (moins de $2.5 \%$ de la longueur développée des versants calcaires) et de très faible volume unitaire.

\subsection{Stabilité des marnes}

\subsection{Etat actuel}

Comme on l'a vu dans le paragraphe précédent, les pentes des versants marneux sont douces, presque toutes inférieures à $20^{\circ}$. Cependant, sur les $134 \mathrm{~km}$ de longueur développée de la retenue, une cinquantaine de glissements sont visibles (répartis dans douze des vingt zones recensées), certains anciens et stabilisés, d'autres récents et actifs.

Il faut distinguer :

- des glissements superficiels, du type « slab-slide " d'après la classification de Skempton, dont la profondeur atteint quelques mètres, une dizaine au maximum. C'est la grande majorité des glissements observés. Les pressions d'écoulement de l'eau dans les couches superficielles à la suite de fortes pluies jouent sans doute un rôle prépondérant dans leur mise en mouvement ( $800 \mathrm{~mm}$ de pluie en six mois) ;

- des glissements rotationnels profonds. Ils sont en nombre limité. Seuls quelques glissements (8) se rattachent de façon nette à ce type.

Les volumes des glissements observés sont difficiles à évaluer, d'une part à cause de l'échelle réduite du plan topographique disponible, d'autre part à cause de la difficulté d'estimer la profondeur de ces glissements. On peut cependant avancer les points suivants, les volumes cités ne constituant qu'un ordre de grandeur

- il n'existe que trois glissements mettant en jeu des volumes relativement importants (de plusieurs centaines de milliers de $\mathrm{m}^{3}$ ), le maximum qui correspond au "glissement des oliviers " (zone $\mathrm{n}^{\circ} 1$ ); qui sera examiné plus tard en détail, devant se situer entre 500000 et $1000000 \mathrm{~m}^{3}$;

- trois autres glissements ont un volume atteignant $100000 \mathrm{~m}^{3}$;

- la majorité des glissements met en jeu des volumes plus faibles, de l'ordre de $10000 \mathrm{~m}^{3}$ et moins.
Le volume total des glissements observés est de quelques millions de $\mathrm{m}^{3}, 5$ millions de $\mathrm{m}^{3}$ constituant très certainement un maximum.

Les pentes des quelques glissements suffisamment importants pour être repérés sans erreur grossière sur le plan topographique, ont été reportées sur le graphique 6. On constate que les glissements apparaissent pour des pentes supérieures à $15^{\circ}$ environ.

Pour un matériau de caractéristiques mécaniques données, il existe un profil de talus, tel qu'en tous points de ce talus l'équilibre limite soit atteint. En traçant ce profil pour différents couples cohésion-angle de frottement (pour un poids volumique de $20 \mathrm{kN} / \mathrm{m}^{3}$ ), on a recherché celui qui s'ajustait le mieux à l'ensemble des points portés sur le graphique. Le profil retenu est indiqué sur ce graphique ; il correspond à $\Phi=15^{\circ}$ et $\mathrm{C}=30 \mathrm{kN} / \mathrm{m}^{2}$. Les caractéristiques mécaniques ainsi déterminées ne sont pas les caractéristiques réelles des marnes, mais des caractéristiques apparentes moyennes, tenant compte implicitement de l'influence complexe de l'écoulement de l'eau dans le talus, de la fissuration éventuelle des marnes, etc.

\subsection{Evolution éventuelle}

Par suite de la mise en eau et des variations de niveau du plan d'eau, la stabilité des versants peut être affectée :

- par une modification des caractéristiques mécaniques des marnes;

- par une modification des efforts sollicitant ces versants.

D'après les prélèvements effectués à l'amont de la retenue aux environs de Matmata par le laboratoire de Casablanca, les marnes du trias et du miocène. sont pratiquement saturées à très faible profondeur, une diminution des caractéristiques mécaniques qu'entraînerait une augmentation de la teneur en eau ne semble donc pas à craindre.

En ce qui concerne les effets de la retenue, il faut considérer d'une part l'effet de déjaugeage de la partie inférieure du talus jusqu'à un niveau critique du plan d'eau en fonction de la hauteur du versant, d'autre part et surtout l'effet de la vidange rapide du réservoir (vitesse moyenne $=1.5 \cdot 10^{-5} \mathrm{~m} / \mathrm{s}$ ), les pressions interstielles ne pouvant se dissiper dans ces marnes imperméables, même lorsqu'elles sont fissurées $\left(\mathrm{K} \leqslant 10^{-7} \mathrm{~m} / \mathrm{s}\right)$. 


\subsection{Glissements superficiels}

L'équilibre du talus indéfini à une profondeur $z$ peut être apprécié pour un écoulement parallèle au talus par l'équation

$$
\frac{C^{\prime}}{\gamma z}=-\frac{1}{2} \cdot \sin 2 \theta-\frac{\gamma}{\gamma^{\prime}} \cdot \cos ^{2} \theta \cdot \operatorname{tg} \Phi^{\prime}
$$

$\gamma^{\prime}$ : poids volumique submergé ;

$\gamma$ : poids volumique saturé ;

$\theta$ : angle du talus sur l'horizontale ;

$C^{\prime}$ et $\Phi^{\prime}$ : caractéristiques effectives des marnes.

Le graphique 7 montre qu'avec les caractéristiques (en pointe) mesurées en profondeur $(z>10 \mathrm{~m})$ la stabilité est assurée pour des pentes atteignant $25^{\circ}$ à $28^{\circ}$. Par contre, les caractéristiques statistiques tirées des glissements observés ( $\mathrm{si}$ on les assimile à des caractéristiques effectives) conduisent à un équilibre pour les talus de pente inférieure à $15^{\circ}$ et $16^{\circ}$.

\subsection{Glissements profonds}

L'utilisation des abaques de Hoek ( $\left.{ }^{1}\right)$ au cas " moyen " comportant un talus de $100 \mathrm{~m}$ de hauteur (sans fissuration), submergé sur sa moitié inférieure et soumis à une vidange rapide donne l'équilibre pour les valeurs suivantes (en rupture circulaire) :

$$
\begin{array}{lll}
\beta=25^{\circ} & C^{\prime}=65 \mathrm{kN} / \mathrm{m}^{2} & \Phi^{\prime}=17^{\circ} \\
\beta=20^{\circ} & C^{\prime}=45 \mathrm{kN} / \mathrm{m}^{2} & \Phi^{\prime}=17^{\circ} \\
\beta=15^{\circ} & C^{\prime}=25 \mathrm{kN} / \mathrm{m}^{2} & \Phi^{\prime}=17^{\circ}
\end{array}
$$

Rappelons que les valeurs mesurées par le laboratoire de Casablanca sur les marnes de la cuvette sont $\Phi=15^{\circ}$ et $C^{\prime}=60 \mathrm{kN} / \mathrm{m}^{2}$, valeurs obtenues sur des échantillons situés à une protondeur relativement faible (10 $\mathrm{m}$ environ). Toutefois, il s'agit de caractéristiques de post-rupture mais non de caractéristiques résiduelles, encore que ces dernières ne soient à considérer que dans le cas d'une surface de cisaillement pré-existante (ancien glissement) ou dans des zones fissurées. En dehors de ces cas particuliers, les caractéristiques des couches plus profondes sont sans doute meilleures.

Notons que les cercles de rupture correspondant au cas " moyen " ci-dessus pénètrent de 50 à $60 \mathrm{~m}$ dans les marnes et qu'il ne devrait donc pas y avoir de rupture profonde sauf dans le cas d'une surface de cisaillement (charriage, faille) ou d'un ancien glissement réduisant à $17^{\circ}$ ou $15^{\circ}$ la valeur résiduelle du cisaillement.

\subsection{Volume maximal de l'ensemble des glissements éventuels}

Par suite de la mise en eau, et surtout en cas de vidange rapide de la retenue, les glissements actuels risquent d'être remis en mouvement. De nouveaux glissements pourront apparaître. Les considérations précédentes montrent cependant que la mise en mouvement de volumes importants n'est pas à craindre, et que les glissements seront généralement superficiels et progressifs.

Nous avons vu que les glissements s'observaient pour des pentes supérieures à $15^{\circ}$ à $20^{\circ}$. Les versants marneux de pentes supérieures à $15^{\circ}$ représentent $15 \%$ environ de la retenue, soit $20 \mathrm{~km}$ de longueur. Pour

(1) Voir Rock Slope Stability on Open Mining - Dr. E. Hoek, Imperial College - Rock Mechanics Report No. 4 unpublished, July 1970.

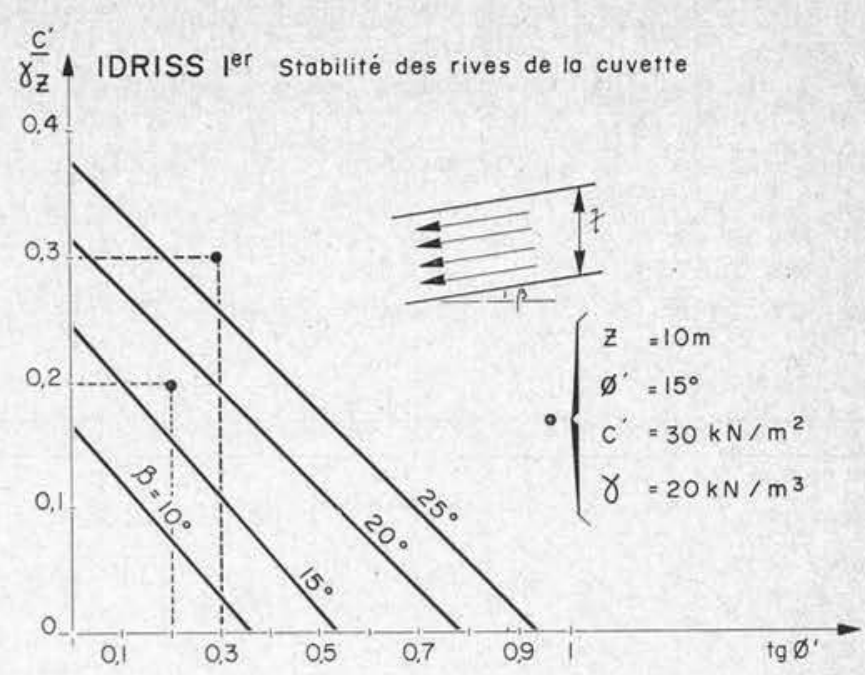

Fig. 7.

une hauteur moyenne de $100 \mathrm{~m}$, la surface totale des glissements potentiels est de 6 millions de $\mathrm{m}^{2}$. Une profondeur moyenne de glissement de $10 \mathrm{~m}$ conduit à un volume global de 60 millions de $\mathrm{m}^{3}$, soit environ $5 \%$ du volume total de la cuvette. Encore faut-il noter que plus de la moitié de ce volume est déjà situé dans la cuvette et que la réduction de capacité n'excèdera probablement pas 2 à $3 \%$. Ce chiffre est à considérer avec beaucoup de prudence, compte tenu des hypothèses extrêmes faites mais il constitue très certainement le maximum du volume total susceptible de glisser progressivement à très long terme dans la cuvette. Il ne faut pas oublier par ailleurs qu'il s'agirait dans ce cas d'une multitude de petits glissements dont le volume individuel le plus important n'excèderait pas un million de $\mathrm{m}^{3}$ (voir plus loin paragraphe 6.1 ).

\subsection{Stabilité des calcaires et calcaires marneux}

L'écroulement en masse des calcaires, par la rapidité avec laquelle ces masses rocheuses sont susceptibles de se déplacer, pourrait créer une vague dangereuse dans la retenue si les volumes instables impliqués étaient importants.

La stabilité des calcaires est fonction d'un certain nombre de facteurs tels que :

- l'importance du massif calcaire et de son enracinement ;

- la disposition favorable ou non des bancs;

- la fracturation en grandes masses par des failles;

- l'existence possible avec inclinaison vers la vallée soit de lits marneux entre les bancs calcaires, soit de surfaces de cisaillement recoupant tout le massif ;

- la partie inférieure plus marneuse du versant.

On retrouve certains de ces facteurs dans les calcaires de la cuvette (fig. 1).

- dans les zones $\mathrm{n}^{\circ} 2$ et 4 la conjugaison de certains d'entre eux a provoqué le mouvement de masses relativement importantes $\left(300000 \mathrm{~m}^{3}\right.$ au total) encore que sans conséquence pour une retenue de plus de un milliard de $\mathrm{m}^{3}$ ) ;

- les zones no 9 et 11 paraissent actuellement stables bien que les bancs soient inclinés vers la vallée (pendage de $45^{\circ}$ pour la zone $n^{\circ} 9$ et $20^{\circ}$ pour la zone $\left.\mathrm{n}^{\circ} 11\right)$; 
- dans la zone $n^{\circ} 1$ le massif calcaire apparemment mince est actuellement stable bien qu'il empêche les marnes du complexe situées derrière lui de se répandre dans la vallée de l'oued Inaouène ;

- ailleurs notamment en rive droite juste à l'amont du barrage, le massif calcaire donne tous les signes extérieurs de stabilité. Les bancs y plongent en effet vers la montagne, avec cependant en un point très localisé une faible composante vers la vallée, les accidents qui découpent le massif sont proches de la verticale.

Après remplissage de la retenue à la cote maximale (220), on peut envisager que :

- les masses des zones no 2 et 4 pourront s'affaisser davantage dans la retenue, entraînant peut-être quelques écroulements de rochers situés de part et d'autre, sans aucun caractère de gravité pour la zone 4 (quelques milliers de $\mathrm{m}^{3}$ ), mais qui pourraient avoir plus d'ampleur pour la zone 2 (quelques centaines de milliers de $\mathrm{m}^{3}$, voir plus loin paragraphe 6.2 ) ;

des cisaillements pourront se produire dans les joints marneux éventuels entre les bancs calcaires des zones $\mathrm{n}^{\circ} 9$ et 11 , menaçant ainsi les douars proches du versant;

- l'hypothèse d'un cisaillement dans la masse de l'éperon calcaire de la zone $\mathrm{n}^{\circ} 1$, sous la poussée des marnes du complexe n'est pas à rejeter $a$ priori (voir plus loin paragraphe 6.1 ) ;

- dans la retenue, aucun plan de cisaillement éventuel tapissé de matériaux marneux, incliné vers la vallée et recoupant en écharpe les massifs stables de la rive droite n'a été noté, mais l'existence de tels plans ne peut pas non plus être entièrement masquée par les basses et moyennes terrasses. De tels cisaillements ont été notés à $600 \mathrm{~m}$ à l'aval du site dans les calcaires de la rive droite.

\subsection{Couverture de pente - matériaux d'altération}

Au cours de la mise en eau, le déjaugeage du pied des talus contribue à diminuer leur stabilité. Si ceux-ci se trouvent actuellement en équilibre limite, la mise en eau peut conduire à des déplacements de ces pentes, sans conduire nécessairement à des glissements. En effet, une faible rotation de la masse en mouvement permet de rétablir l'équilibre. Une mise en eau progressive se traduit alors par des mouvements assez faibles (1) de ces matériaux sans glissements d'ensemble importants. En fait, la plupart des glissements observés depuis la mise en eau (trois remplissages et vidanges successifs) affectent ces terrains argileux quaternaires dans trois des vingt zones étudiées. Des crevasses importantes sont apparues, mais jusqu'alors sans déplacement de masse et les volumes concernés sont restés faibles.

\section{ETUDE DETAILLEE DES ZONES PARTICULIERES}

Dix-neuf zones de glissements et d'éboulements ont été inventoriées (voir fig. 1 et tableau 2 ci-après) suivant le type de giissement superficiel ou profond, la nature du matériau, la pente moyenne du talus, la hauteur totale et la hauteur au-dessus des plus hautes eaux, la longueur de versant concernée, la distance au barrage, le volume de matériaux impliqué.

A la suite de ce classement, trois zones seulement ont fait l'objet d'études vraiment détaillées; les zones 1 et 2 , les plus importantes en volume, situées près du barrage et les zones 6 et 7 concernant la voie ferrée Fès-Oujda.

\subsection{Zone située dans le complexe marneux}

C'est la zone 1 dite " glissement des oliviers ", située en rive gauche à environ $0.5 \mathrm{~km}$ du barrage; elle s'étend sur environ $500 \mathrm{~m}$ et est caractérisée par trois cuillères de glissements successives; la première allant des cotes (170) à $(220)$ avec un front à la base de $200 \mathrm{~m}$, est la plus importante; la seconde est située au-dessus de (220) et la troisième, beaucoup plus pincée, remonte à l'heure actuelle jusqu'à la cote $(300)$ environ. Ces glissements évoluent régulièrement et continuellement. Cette évolution est facilitée par la présence permanente d'eau qui ressort en un petit filet à la cote (225) environ, sur le bord amont du glissement.

La zone I est butée en pied par un éperon calcaire et on pouvait craindre que cet éperon soit trop mince pour s'opposer à un glissement profond (30 à $40 \mathrm{~m}$ )

(1) Voir \&The dynamics of a landslide produced by filling a reservoir».

H. Breth, $9^{\circ}$ Congrès des Grands Barrages, Istambul. Q. 32 R3 - Vol. I, pp 37 (1967). qui aurait pu intéresser un volume très important (fig. 8 et 9) de l'ordre du million de $\mathrm{m}^{3}$. En fait, un levé géologique au $1 / 20000$, trois sondages et des tranchées ont permis d'éliminer ce risque.

Interposé entre le complexe marneux situé dans le versant gauche de la vallée et le lit de l'oued Inaouène, cet éperon se comporte comme un mur de soutènement auto-stable dans les conditions hydrogéologiques actuelles.

Les travaux de reconnaissance ont permis de préciser sa géométrie (fig. 10). Montant presque partout audessus des plus hautes eaux de retenue de deuxième phase à (220.30), cet éperon est en réalité un véritable barrage naturel haut de 60 à $70 \mathrm{~m}$ et large d'au moins $250 \mathrm{~m}$ à la cote de loued dans le profil I et de $150 \mathrm{~m}$ dans le profil II plus amont. L'empattement de ce barrage naturel est donc suffisant pour garantir sa stabilité après remplissage de la retenue.

De plus, les bancs plongent vers l'intérieur de la rive de 15 à $20^{\circ}$ sur l'horizontale et aucun niveau véritablement argileux n'a été repéré en sondage. On ajoutera que d'après les carottes, le rocher est peu fissuré mais que les fissures sont tapissées de gypse et oxydées presque jusqu'au niveau de l'oued. Ces indices indéniables de circulation d'eau sont confirmés par les pertes totales d'eau de perforation dès l'entrée des forages dans les calcaires. On pouvait done penser qu'en cas de vidange rapide, le calcaire se comporterait comme un massif auto-drainant et auto-stable.

L'exploitation de la retenue au niveau 203 n'a pas provoqué de mouvement d'ensemble, des crevasses nouvelles sont apparues récemment vers la cote 225 , mais elles sont en rapport avec les sources qui sortent en ces points et non avec le marnage de la retenue. 
IDRISS ler Stabilité des rives de la cuvette

TABLEAU N $N^{\circ} 2$ - INVENTAIRE DES ZONES DE GLISSEMENTS ET

D. EBOULEMENTS SUR LES RIVES DE LA CUVETTE

\begin{tabular}{|c|c|c|c|c|c|c|c|c|c|c|c|c|c|}
\hline \multirow[b]{2}{*}{$\begin{array}{l}0 \\
0 \\
\vdots \\
0 \\
N\end{array}$} & \multirow[b]{2}{*}{ 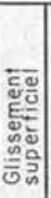 } & \multirow[b]{2}{*}{ 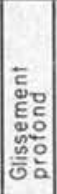 } & \multirow[b]{2}{*}{ 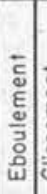 } & \multirow[b]{2}{*}{ 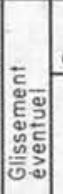 } & \multicolumn{3}{|c|}{$\begin{array}{l}\text { Noture } \\
\text { du matériou }\end{array}$} & \multirow{2}{*}{ 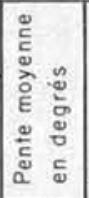 } & \multirow{2}{*}{ 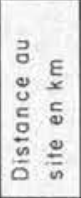 } & \multirow{2}{*}{ 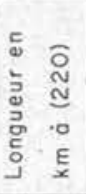 } & \multirow[b]{2}{*}{ 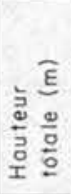 } & \multirow{2}{*}{ 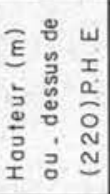 } & \multirow{2}{*}{ 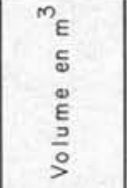 } \\
\hline & & & & & 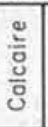 & 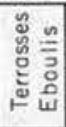 & \begin{tabular}{|l|} 
\\
$\vdots$ \\
$\vdots$ \\
$\vdots$ \\
$\vdots$ \\
$\Sigma$ \\
$\Sigma$
\end{tabular} & & & & & & \\
\hline 1 & & & & & & & & 20 & 0,5 & 0,20 & 130 & 80 & $>500.000$ \\
\hline 2 & & & & & & & & 29 & 1,2 & 0,15 & 120 & 70 & $<500000$ \\
\hline 3 & & & & & & & & 12 & $\begin{array}{l}2,0 \\
\text { R.O }\end{array}$ & 0,15 & 130 & 80 & 100.000 \\
\hline 4 & & & & & & & & 24 & 1,8 & 0,24 & 90 & 50 & 100.000 \\
\hline 5 & & & & & & & & \begin{tabular}{|l|}
8.10 \\
10.15 \\
\end{tabular} & $\begin{array}{r}4,5 \\
R .6 \\
\end{array}$ & $\begin{array}{l}0.70 \\
1.00\end{array}$ & \begin{tabular}{|c|}
100 \\
80 \\
\end{tabular} & $\begin{array}{l}50 \\
50 \\
\end{array}$ & 100.000 \\
\hline 6 & & & & & & & & 7010 & 13.0 & 1,50 & 40 & 20 & 10.000 \\
\hline 8 & & & & & & & & 23 & 8,0 & & 30 & 0 & \\
\hline 9 & & & & & & & & 27040 & $\begin{array}{l}3,0 \\
R . G\end{array}$ & 1,80 & 40 & 0 & \\
\hline 10 & & & & & & & & 17 & 6,4 R.D & 0,15 & 30 & -10 & 1.000 \\
\hline 11 & & & & & & & & 22 & 8,2 & $\begin{array}{l}1.00 \\
0.50\end{array}$ & $\begin{array}{l}65 \\
50\end{array}$ & $\begin{array}{l}30 \\
10\end{array}$ & 10.000 \\
\hline 12 & & & & & & & & 11 áı & $9,4 \mathrm{RD}$ & 0,25 & 100 & 86 & 10.000 \\
\hline 13 & & & & & & & & & $\begin{array}{l}14,0 \\
\text { R. } \\
\end{array}$ & 0,05 & 10 & & 1.000 \\
\hline 14 & & & & & & & & 16 oا 19 & 13,0 RD & 0,20 & 50 & 30 & \\
\hline 15 & & & & & & & & & $\begin{array}{c}16,0 \\
\text { RD }\end{array}$ & & & & \\
\hline 16 & & & & & & & & 15 & 18,0 & 0,10 & 40 & 20 & \\
\hline 17 & & & & & & & & & $\begin{array}{r}20,0 \\
\text { R.D } \\
\end{array}$ & & & & \\
\hline 18 & & & & & & & & 27 & $\begin{array}{l}21,0 \\
R D\end{array}$ & 2,00 & 70 & 80 & 200.000 \\
\hline 19 & & & & & & & & 18 ذ24 & 20,0 & 1,40 & 65 & 60 & \\
\hline
\end{tabular}

IDRISS fer Stabilité des rives de la cuvette. GLISSEMENT DES OLIVIERS (ZoneI)

VIDANGE RAPIDE

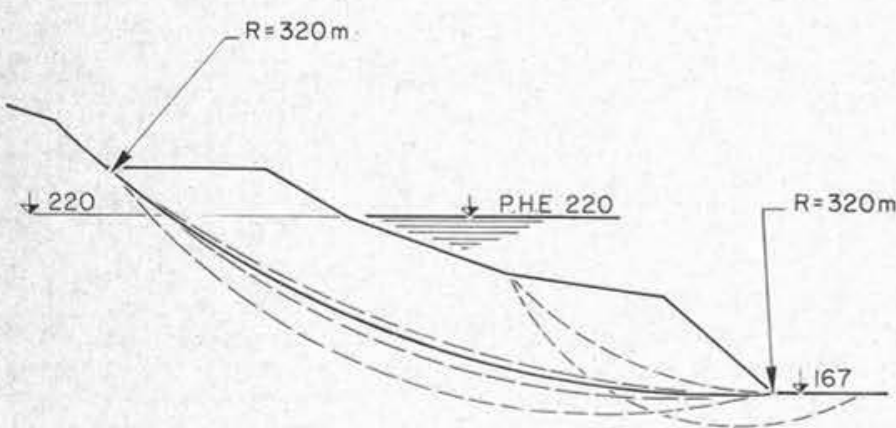

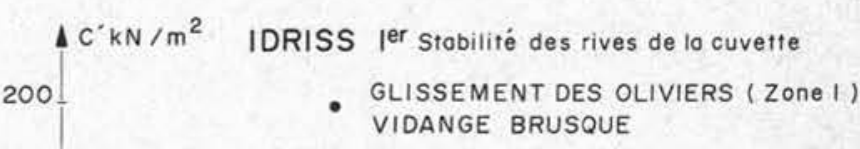
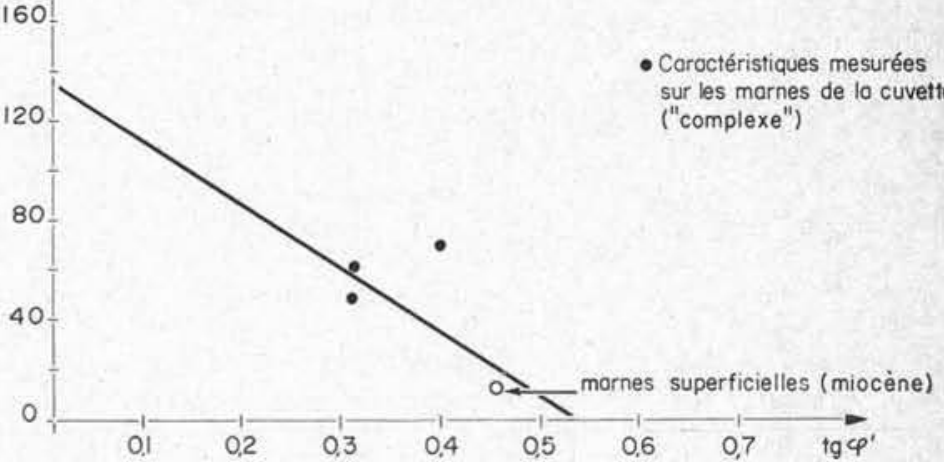

Fig. 9.

Fig. 8.

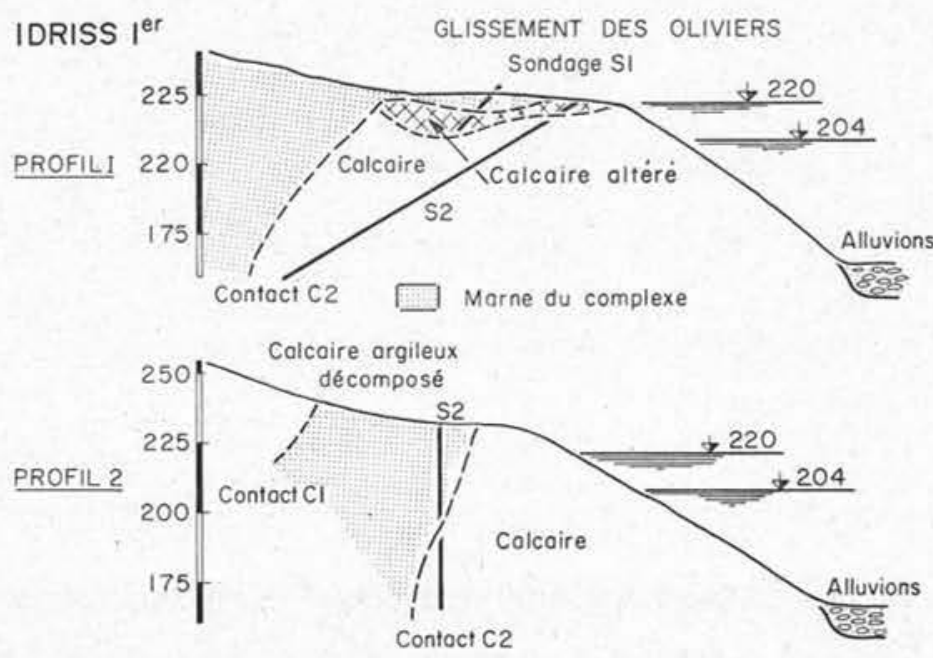

Fig. 10. 


\subsection{Zone située dans les calcaires}

C'est la zone 2 en rive droite, située à environ $1.2 \mathrm{~km}$ en amont du barrage.

Il s'agissait d'un éboulement dans les calcaires reposant sur des marnes grises pentées vers la vallée, démarrant à la cote 295 environ (75 m au-dessus de la retenue).

Une carte géologique détaillée (au $1 / 2000$ ) a été dessinée.

Son examen montre bien l'importance des marnes grises de base dans le déclenchement du mouvement : la talaise supérieure de calcaire détachée par une faille en arrière de la masse déplacée a glissé sur les marnes, en se disloquant plus ou moins. Ces calcaires ont dû jouer un rôle pour amener et amasser de l'eau au toit des marnes saines, des crevasses ayant été détectées en arrière de la surface faillée limitant la masse déplacée.

On ne voit pas les bourrelets caractéristiques du bas d'un glissement qui affecterait la totalité de la masse marneuse avec sortie du cercle de glissement, soit au pied du versant, soit même au-delà.

On peut donc penser que le mouvement s'est produit au toit des marnes saines, compactes et donc imperméables, les marnes altérées sus-jacentes étant ellesmêmes très probablement plus perméables ; et ceci plus par une sorte de translation que de rotation.

On a donc, en définitive, un mouvement qui ne correspond ni à un écroulement rocheux, toujours rapide et brutal, ce que l'on pouvait craindre, ni au glissement argileux type.

On peut penser cependant qu'il doit avoir la particularité d'être un mouvement relativement lent et il n'y a aucune crainte à avoir en ce qui concerne le barrage, le volume global des masses susceptibles de se mettre en mouvement, évalué d'après le levé au $1 / 2000$, n'excédant pas $500000 \mathrm{~m}^{3}$. Là encore, on doit seulement aboutir à un adoucissement du talus qui sera en même temps et dans une certaine mesure, buté finalement au pied par les zones ébouleuses à blocs calcaires.

En fait, depuis trois ans d'exploitation, divers mouvements se sont produits, mais seule la partie inférieure aval présente une crevasse récente, le reste n'a pas encore été remis en mouvement. Par contre, à l'amont et à l'aval, des crevasses en chevron se forment qui affectent surtout les crêtes plus ou moins couvertes d'éboulis situées entre les petits ravins; mais les volumes unitaires intéressés restent très faibles et ne peuvent provoquer de vagues dangereuses.

\subsection{Stabilisation de la voie ferrée Fès-Oujda}

La voie ferrée ne sera déviée que lors de la $2^{\mathrm{e}}$ phase de l'exploitation du barrage (sur $15 \mathrm{~km}$ environ). Ce projet de déviation a été étudié par l'Office National des Chemins de Fer avec le concours du Laboratoire Public d'Etudes et d'Essais de Casablanca. Lors de la première phase de mise en eau, la voie ferrée située 10 à $15 \mathrm{~m}$ au-dessus de la retenue est très exposée en quatre points. Ces zones ont été reconnues à l'aide de puits (de $15 \mathrm{~m}$ de profondeur) permettant de prélever des échantillons intacts dans les marnes miocènes plus ou moins altérées et fissurées, de sondages pénétrométriques et piézométriques, de forages à la tarière en pied de talus dans les alluvions.

Un schéma des recharges de confortement (zone 6-D) fut étudié et proposé par Coyne et Bellier, les carac- téristiques définitives des différents ouvrages confortatifs des quatre zones ayant été étudiées par le Laboratoire Public d'Etudes et d'Essais de Casablanca. Il ne sera ici question que de la zone 6-D.

Dans cette zone, la marne altérée sur 5 à $10 \mathrm{~m}$ d'épaisseur montre de nombreux glissements, en coulées de pente apparemment de faible épaisseur. Elle surmonte les marnes oxydées beiges compactes puis à 10 ou $15 \mathrm{~m}$ de profondeur les marnes gris-bleu compactes, toutes ces marnes sont fissurées et plastiques (indice de plasticité de 30 à $50 \%$, plus faible en profondeur). Les marnes glissées superficielles semblent recouvrir les alluvions d'oued à la cote (182-183) en pied de talus, recouvertes elles-mêmes par les limons. La cote de la voie ferrée est voisine de (218-219) et la hauteur du talus est de l'ordre de $25 \mathrm{~m}$ (fig. 12).

En ce qui concerne la piézométrie, la nappe voisine de la cote $(186.00)$ en pied de talus remonte de façon plus ou moins irrégulière sous la pente à 5 ou $10 \mathrm{~m}$ au-dessous du terrain naturel, approximativement suivant le toit des marnes beiges compactes.

Les caractéristiques mécaniques des marnes altérées et saines ont été déterminées sur des prélèvements intacts, soit à l'appareil de cisaillement direct, soit à l'appareil triaxial. Les déformations maximales obtenues au laboratoire n'ont pas excédé 6 à $8 \%$ et il est probable que les caractéristiques préconisées et qui correspondent à ces déformations $\left(C^{\prime}=10 \mathrm{kN} / \mathrm{m}^{2}\right.$, $\Phi=25^{\circ}$ ) ne représentent pas les valeurs résiduelles (sans doute voisines, compte tenu des indices de plasticité élevés, 30 à $50 \%$, de $C_{r}=0, \Phi_{r}=20^{\circ}$ ), qui ne pouvaient être mesurées que par des essais à très fortes déformations (cisaillements alternés ou cisaillements par torsion). La prise en compte de ces valeurs résiduelles serait toutefois par trop pessimiste dans ce cas, les valeurs recommandées par le projeteur correspondant sensiblement aux valeurs minimales assurant la stabilité des pentes dans les conditions présentes avant mise en eau (talus moyen de 2 horizontal pour 1 vertical)

Il s'agissait d'étudier un confortement permettant de stabiliser ce talus de marnes qui sera soumis aux effets du marnage du réservoir (203 retenue normale 206 plus hautes eaux exceptionnelles, vidange totale jusqu'à 180). La solution retenue, la seule à l'échelle du problème a consisté à disposer une risberme drainante en enrochement compacté au pied du talus, sur environ $570 \mathrm{~m}$ de longueur.

La stabilité est assurée avec un coefficient de sécurité normal (1.20 à 1.25$)$ pour les hypothèses de vidange rapide (vitesse supérieure à $2 \mathrm{~m}$ /jour), les plus vraisemblables, c'est-à-dire depuis la cote (203) jusqu'au pied du talus (fig. 11).

En effet, il a été considéré qu'il serait excessif de se protéger, avec le même degré de sécurité, contre une vidange depuis le niveau des plus hautes eaux (206.75) correspondant à la crue du projet de période de retour supérieure à mille ans.

En fait, pour les plus fortes crues observées au cours des douze dernières années, le niveau n'aurait dépassé la cote (203) que pendant moins d'un jour et sans excéder la cote (203.60) et le niveau de crue centennale n'aurait dépassé la retenue normale que pendant moins de quatre jours et sans excéder (204.50).

Dans ces conditions, il a paru normal d'accepter un coefficient de sécurité limite (1.05) pour le cas de la vidange maximale depuis la cote des plus hautes eaux (206), le temps de submersion au-dessus de (203) étant trop court pour que la ligne de saturation à (206) 
IDRISS jer Stabilité des rives de la cuvette STABILISATION DE LA VOIE FERREE FEZ . OUJDA

Zone 6.D

\begin{tabular}{|l|c|c|c|}
\hline Yidanges rapides & Risberme 2 & Risberme 4 & Talus naturel \\
\hline 206 à 192 & 1,25 & 1,08 & 0,77 \\
\hline 203 à 192 & 1,33 & 1,20 & 0,86 \\
\hline
\end{tabular}

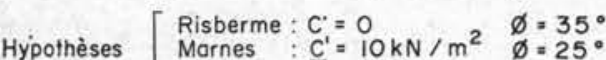

Vidange : $\overline{\mathrm{B}}=1,00$

RISBERME 2

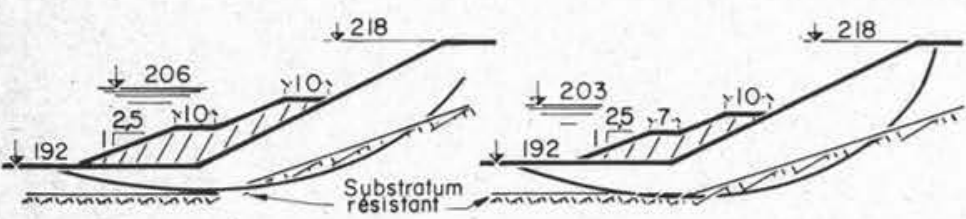

Fig. 11 .

s'établisse dans le terrain. Bien entendu, la sécurité ne serait plus assurée en cas de forte secousse sismique survenant juste après une vidange, même partielle. Les recharges de confortement proposées résultent donc du choix raisonnable entre un projet très conservatif basé sur les caractéristiques de cisaillement résiduelles (cohésion nulle notamment), sur les conditions aux limites les plus défavorables et sur les hypothèses de pressions interstitielles les plus pessimistes, projet qui serait par suite très coûteux, et un projet d'un prix acceptable ne faisant subsister que des risques relativement faibles mais pouvant nécessiter des consignes d'exploitation particulières et un entretien plus continu dans les zones incriminées. Ce choix apparaît tout à fait justifié sous réserve, d'une part, d'observer régulièrement le comportement de la voie
IDRISS jer Stabilité des rives de lo cuvette VOIE FERREE FEZ OUJDA

Zone D. Profil type

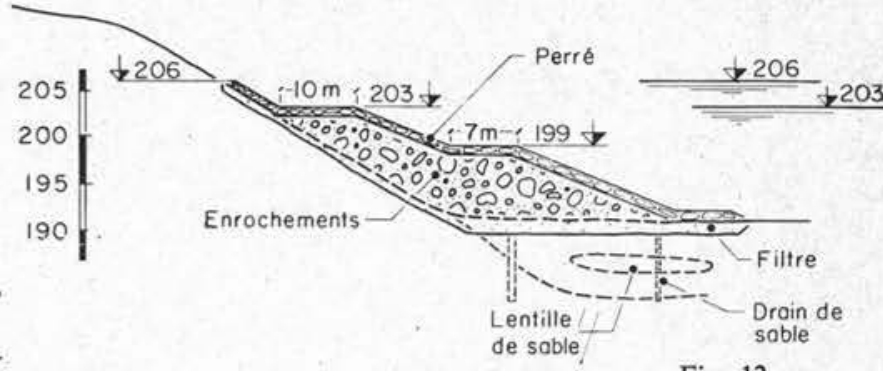

Fig. 12.

filtre tout venant et protégé par un perré contre le batillage. Par mesure de précaution, le projeteur a prévu en outre deux lignes de drains de sable sous le pied de la recharge pour décomprimer les souspressions éventuelles qui pourraient subsister après vidange de la retenue dans des lentilles ou couches d'alluvions plus ou moins perméables. Sans apparaître indispensables compte tenu de ce que l'on sait de la géologie de la fondation, ces puits apportent une certaine sécurité supplémentaire au prix d'une dépense relativement faible.

\subsection{Glissements menaçant les villages ou fermes}

Enfin, une attention particulière a été portée à l'étude des glissements pouvant menacer des douars, villages ou fermes.

IDRISS $1^{\text {er }}$ Stabilité des rives de la cuvette

TABLEAU N $\mathrm{N}^{\circ} 3$ - DOUARS MENACES PAR LES GLISSEMENTS

A LA MISE EN EAU

\begin{tabular}{|c|c|c|c|c|c|c|}
\hline \multirow{2}{*}{ Zones } & \multicolumn{3}{|c|}{ Noture du Substratum } & \multirow{2}{*}{$\left|\begin{array}{l}\text { Douors menocés } \\
\text { ere }{ }^{\text {pre }} \text { (206) }\end{array}\right|$} & \multirow{2}{*}{$\begin{array}{l}\text { Douors noyes } \\
2^{\mathrm{e}} \text { phase }(220)\end{array}$} & \multirow{2}{*}{$\begin{array}{c}\text { Douars menocés } \\
2^{\mathrm{e}} \text { phose (220) }\end{array}$} \\
\hline & Calcaires & Terrasses & Marnes & & & \\
\hline \multicolumn{7}{|l|}{1} \\
\hline \multicolumn{7}{|l|}{3} \\
\hline \multicolumn{7}{|l|}{8} \\
\hline \multicolumn{7}{|l|}{9} \\
\hline \multicolumn{7}{|l|}{11} \\
\hline \multirow{2}{*}{\multicolumn{7}{|c|}{$\frac{13}{15}$}} \\
\hline & & & & & & \\
\hline \multicolumn{7}{|l|}{16} \\
\hline \multicolumn{7}{|l|}{17} \\
\hline \multicolumn{7}{|l|}{18} \\
\hline 20 & & & & & $\vec{x}$ & \\
\hline
\end{tabular}

ferrée (par nivellement entre autres), notamment lorsque le plan d'eau varie entre (198) et (206), d'autre part, d'imposer en cas de crue, des consignes de manœuvre des vannes de vidange telles qu'il n'y ait qu'une submersion de courte durée au-dessus de la retenue normale (203).

La coupe type de la recharge est représentée sur la figure 12. L'enrochement compacté est posé sur un
Dix douars ou parties de douars sont menacés directement par la retenue, soit au cours de son remplissage de première phase $(8)$, soit au cours de celui de deuxième phase. Sept d'entre eux sont situés sur les marnes, recouvertes ou non par des terrasses tandis que trois autres sont construits sur des calcaires dont le pendage des bancs est défavorable (voir tableau 3). 


\section{CONCLUSION}

De cette étude, nous retiendrons que la méthodologie appliquée à l'évaluation de la stabilité des rives de la cuvette du barrage Idriss $\mathrm{I}^{\text {er }}$ a permis de fixer la limite supérieure du volume des glissements à craindre.

En dépit de la longueur des rives concernées (plus de $130 \mathrm{~km}$ ), ce travail a pu être fait assez simplement. Il a consisté en un inventaire détaillé des caractéristiques géométriques et géologiques des versants, en limitant les reconnaissances et essais géotechniques aux zones les plus critiques intéressant les volumes unitaires les plus grands.

On a ainsi pu conclure que :

- des glissements existent sur les rives de la retenue, mais leur volume global (quelques millions de $\mathrm{m}^{3}$ ) est très faible par rapport au volume de la retenue (1280 millions de $\mathrm{m}^{3}$ sous la retenue normale de deuxième phase) ;

- dans les calcaires marneux, des écroulements se sont produits en des points très particuliers mais il est peu probable qu'il s'en produise ailleurs; s'il s'en produisait, ils seraient très localisés et de faible importance;

- dans les marnes, les glissements relativement importants (supérieurs à $100000 \mathrm{~m}^{3}$ ) sont localisés, mais des pentes supérieures à celles pour lesquelles les marnes sont stables actuellement (c'est-à-dire $15^{\circ}$ ou $20^{\circ}$ suivant les conditions locales) pourront donner lieu à de petits glissements ;
- aucun glissement dans les marnes et aucun écroulement dans les calcaires ne semblent susceptibles de se produire à proximité du barrage, qui pourraient menacer celui-ci soit dans sa stabilité par la vague produite, soit dans son exploitation par l'obturation d'ouvrages de prise ;

- la stabilité de la voie ferrée paraît assurée au cours du remplissage de première phase a (206) par la mise en œuvre des remblais de confortement réalisés par l'ONCF et qui répondent à un souci d'économie qui, en contre partie, implique une observation régulière de leur comportement ;

- un certain nombre de villages menacés par des glissements potentiels doivent être évacués.

Après trois années d'exploitation, les observations montrent que :

- les confortements de la voie ferrée se sont bien comportés ;

- des glissements se sont effectivement produits dans six des zones inventoriées, mais ces mouvements rentrent tout à fait dans le cadre de ce qui était prévu et restent même plutôt inférieurs à ce qui était estimé devoir se produire lors de cette première phase d'exploitation:

- de nouveaux glissements se produiront lors de la phase finale de remplissage qui atteindra des zones instables que la retenue n'a pas encore touchées, mais les observations actuelles permettent de penser que le volume des masses concernées restera inférieur aux volumes globaux estimés. 